\title{
Medical genomic approach to early-onset scoliosis
}

\author{
Shin-Young Yim*i(i) \\ The Center for Torticollis, Department of Physical Medicine and Rehabilitation, Ajou University School of Medicine, Suwon, Korea
}

\begin{abstract}
Early onset scoliosis (EOS) describes the onset of scoliosis before the age of 10 years and is associated with significant health risks. Compared to adolescent idiopathic scoliosis, studies on the etiology of EOS in children are more common. EOS includes inhomogeneous groups of patients, and the etiology of EOS may be congenital, neuromuscular, syndromic, or idiopathic. The identification of the molecular etiology underlying patients with EOS has been increasing. This could provide valuable information for optimizing the management and care of these children. In this review, EOS was reviewed from a medical genomic perspective.
\end{abstract}

Key words: Scoliosis, Child, Genomics.

\section{Introduction}

Scoliosis is the most common developmental disorder of the spine and affects approximately $2 \%$ to $3 \%$ of the population [1-4]. Scoliosis is a three-dimensional torsional deformity of the spine and trunk, where the lateral curvature of the spine (Cobb angle) is deviated by at least $10^{\circ}$ in the coronal plane. Scoliosis is often associated with a rotatory component that produces asymmetry of the rib cage and flank muscles. Disturbance of the sagittal plane is often found, where scoliosis causes the sagittal spinal curvatures (thoracic kyphosis and lumbar lordosis) in the direction of a flat back (Fig. 1).

Scoliosis can develop during infancy or early childhood. Early onset scoliosis (EOS) can be classified into two types depending on the age of onset. Infantile scoliosis is diagnosed in patients aged three years or younger, and juvenile scoliosis is diagnosed in patients aged 4 to 10 years [4-6]. However, the true prevalence of EOS remains unknown. It is estimated that approxi- mately $5 \%$ of fetuses have vertebral anomalies. Approximately $3 \%$ of the normal population has one or two more than the normal seven cervical, 12 thoracic, 5 lumbar, and 5 sacral vertebrae. While, approximately $2 \%$ of the population has one lesser than the normal number of vertebrae $[7,8]$. Considering this, the prevalence of EOS could be higher than speculated. Adolescent idiopathic scoliosis (AIS), affecting people in 10 to 18 years age group, is the most common type of scoliosis [3].

There are two major differences between the EOS and AIS. First, mortality is significantly increased in patients with EOS, but not in AIS [9]. EOS is a potentially a fatal disorder. Young children with EOS are at risk of impaired pulmonary function due to the high risk of progressive spinal deformity and thoracic constraints during the critical period of lung development [4]. The risk of spinal deformity affecting pulmonary development and function is higher in younger children [4]. Severe scoliosis can result in pulmonary restriction caused by three-dimensional distortion of the chest wall, which decreases lung and thoracic volume

Received: 15 November 2021, Accepted: 29 November 2021, Published: 31 December 2021

*Corresponding author: Shin-Young Yim, M.D., Ph.D. (iD https://orcid.org/0000-0002-8521-6331

The Center for Torticollis, Department of Physical Medicine and Rehabilitation, Ajou University School of Medicine, 164 World cup-ro, Yeongtong-gu, Suwon 16499, Korea.

Tel: +82-31-219-5284, Fax: +82-31-219-5209, E-mail: syyim@ajou.ac.kr

Conflict of interest: I declare that I do not have any conflicts of interests.

(c) This is an open-access article distributed under the terms of the Creative Commons Attribution Non-Commercial License (http://creativecommons.org/licenses/by-nc/4.0/) which permits unrestricted non-commercial use, distribution, and reproduction in any medium, provided the original work is properly cited.

(c) Copyright 2021 by the Korean Society of Medical Genetics and Genomics www.e-kjgm.org 


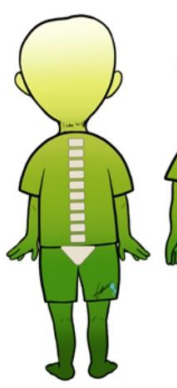

A

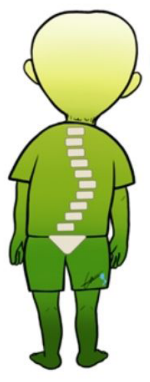

B

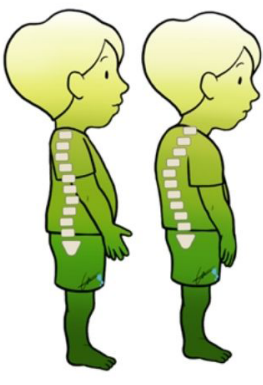

$\mathrm{C}$

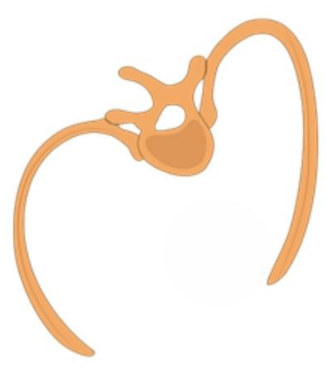

E

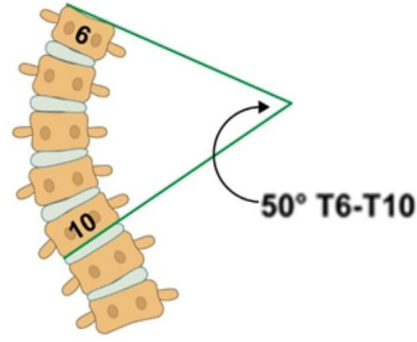

F

Fig. 1. Scoliosis shows three-dimensional torsional deformity of the spine and trunk. Normal spine (A) and spine with lateral curvature higher than $10^{\circ}$ deviation (B) in the coronal plane. Normal spine (C) and kyphosis of the spine (D) on the sagittal plane. (E) Rotation of the vertebrae producing the rib cage and flank muscle asymmetry. (F) Cobb angle showing $50^{\circ}$ in the coronal plane.

and causes abnormal respiratory mechanics $[4,10]$. Thoracic insufficiency syndrome, predominantly in EOS patients younger than five years of age, is among the most severe conditions of pulmonary function. In thoracic insufficiency syndrome, where chest wall deformity interferes with normal lung function and development, symptoms include shortness of breath, fast and shallow breathing during rest, resulting in disrupted sleep, which worsens over time [5]. Unlike EOS, a 50-year follow-up study showed that untreated patients with AIS are still productive and functional at a high level at the 50-year follow-up. Untreated AIS causes little physical impairment other than back pain and cosmetic concerns [11].

Second, the etiology and pathogenesis of AIS remain elusive, while the multifactorial cascade is one of the proposed concepts for the pathogenesis of AIS [12]. This suggests that the AIS originates in the embryonic stages of life from cell types, including adipocytes, osteoblasts, and myoblasts. The involvement of cell types from different developmental origins suggests an environmental process acting in embryonic life at a similar time. It also proposed that the leptin body composition effect is linked to the initiation of the asynchronous neuro-osseous growth mechanism, which involves the creation of a neuraxis tether of relative anterior vertebral overgrowth. Other factors such as age and sex-related anatomical variants of vertebral backward tilt, human upright posture, adolescent growth factors, HueterVolkmann effect in vertebrae, and vertebral bone mass abnormalities, may be involved in both the initiation and progression of scoliosis curvatures in combination with the above factors, resulting in AIS [12].

Etiology of EOS is much more commonly identified than that of AIS. EOS includes inhomogeneous groups of patients, and the etiology of EOS may be congenital, neuromuscular, syndromic, or idiopathic $[6,13]$. Congenital scoliosis results from abnormalities in vertebral development (formation and segmentation) in utero. Among children with neuromuscular disorders, scoliosis is common and characterized by restrictive lung disease caused by respiratory muscle weakness, such as progressive muscular dystrophy. EOS can be found in several syndromes, including neurofibromatosis, Noonan syndrome, Prader-Willi syndrome (PWS), and connective tissue disorders, such as Marfan, Beals, and Ehlers-Danlos syndromes, as well as skeletal dysplasia. Further, storage diseases are also associated with an increased risk of EOS. Treatment strategies and duration significantly differ based on both etiology and the amount of anticipated growth remaining [4]. The true prevalence of EOS is unknown, although idiopathic EOS accounts for $<1 \%$ of all scoliosis cases [4].

Diagnostic tools including X-rays, computed tomography, and magnetic resonance imaging could be used for EOS etiologic diagnosis. Computed tomography is one of the most useful radiological tools in diagnosing congenital scoliosis along with magnetic resonance images. A study [14] showed that 24\% of patients with EOS revealed abnormal magnetic resonance imaging results, and the most common conditions were syrinx and tethered cord. Syrinx with Chiari malformation was the most frequent combination of abnormalities [14]. Identification of the molecular etiology underlying patients with EOS could provide valuable information for both clinical management and prenatal screening [15]. Array comparative genomic hybridization and exome sequencing enable the molecular diagnosis and classification of patients with EOS. The identification of the molecular etiology of EOS could provide useful information for diagnosis by imaging [16]. In a Chinese cohort of 447 patients with unrelated EOS, most had congenital scoliosis, neuromuscular scoliosis, and syndromic conditions, while presumed idiopathic 


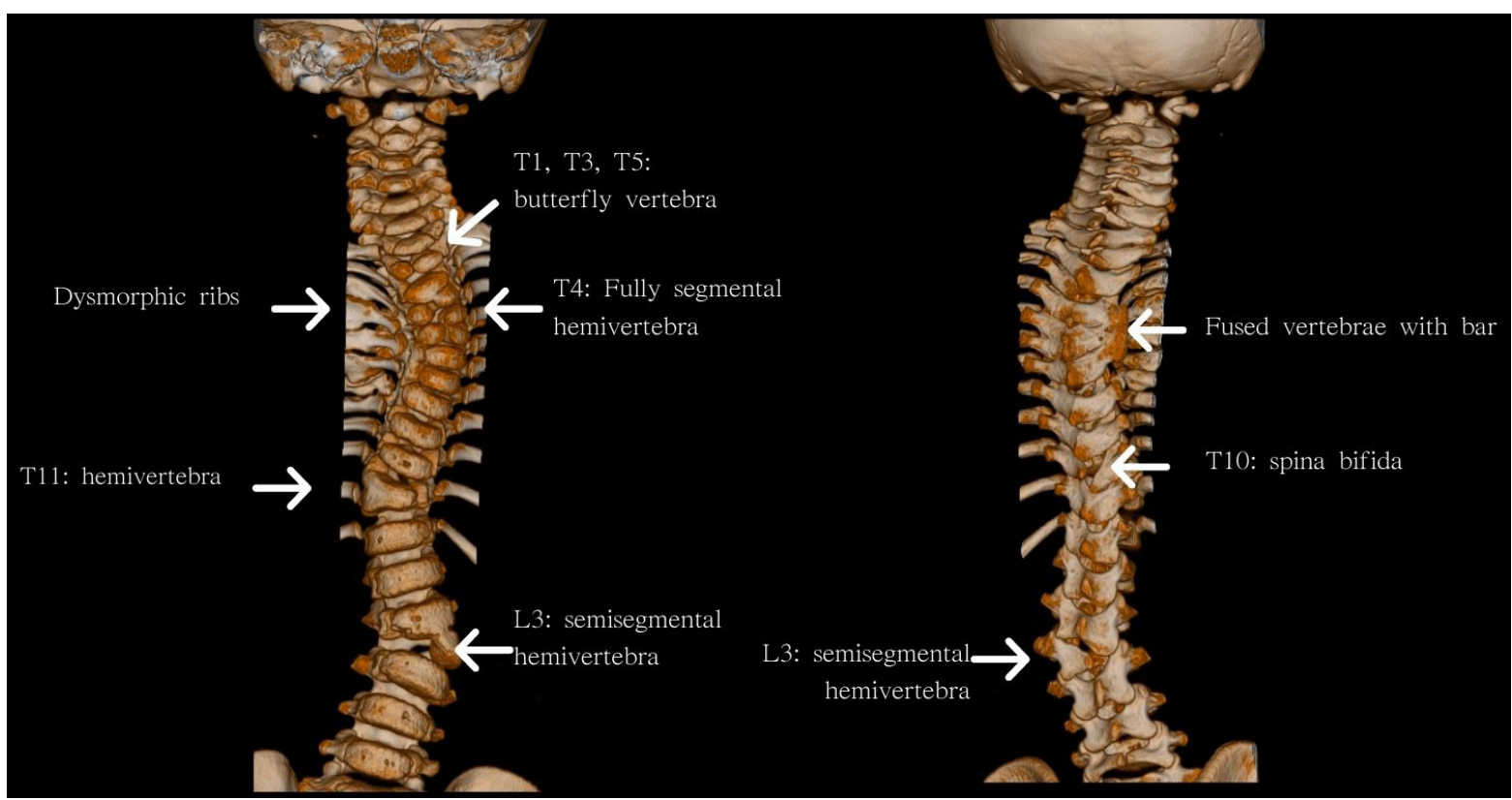

Fig. 2. A 2.4-year-old male with congenital scoliosis. A three-dimensional computed tomogram shows anomalies of vertebral formation and segmentation.

EOS was less common. After exome sequencing data processing and variant interpretation, molecular diagnostic variants were detected in 20.6\% of cases [15].

In this review, EOS is reviewed from a medical genomics perspective.

\section{Congenital Scoliosis}

Congenital scoliosis is a birth defect with variable clinical and anatomical manifestations due to spinal malformation [17]. Spinal malformations result from abnormalities of vertebral formation and/or segmentation in utero, and may include single or multiple hemivertebrae or segmentation defects with or without associated rib fusion (Fig. 2). Congenital scoliosis is often progressive and may require early aggressive treatment. Congenital scoliosis is also often associated with syrinx and tethered cord, as well as abnormalities in other organs. Congenital scoliosis is a relatively common type of EOS. Genetic susceptibility has been implicated in congenital scoliosis, since the embryologic development of the spine is an exquisitely controlled process in a spatio-temporal manner. Deleterious mutations in single genes controlling somitogenesis can lead to rare Mendelian and non-Mendelian disorders affecting spinal integrity [1]. Vertebral formation starts with somitogenesis, which is the process by which somites, blocks of mesoderm that give rise to a variety of connective tissues, are formed. The clock and wavefront model is used to describe the process of somitogenesis in vertebrates. The model describes the splitting of somites from the paraxial mesoderm as a result of the oscillating expression of particular proteins and their gradients $[18,19]$. Somites are epithelialized to form the dermomyotome and sclerotome. Cells of the sclerotome migrate from pairs of paraxial somites and condense either densely or sparsely around the notochord, forming the vertebral bodies and annulus fibrosus, respectively $[1,20]$. In the cartilaginous stage of vertebral development during the sixth week of embryonic development, chondrification centers appear in each mesenchymal vertebra. This develops in a cranial and caudal direction from the cervicothoracic junction. It culminates in approximately the ninth week. The intervertebral disk develops from tissue derived from perinotochordal mesenchymal cells and begins to form in the chondrification stage. Ossification of the cartilaginous elements begins shortly thereafter, and culminates approximately between the 14th and 18th weeks of embryonic development [7]. Any disturbances during this process can lead to congenital scoliosis, in which genetic predisposition plays a role.

\section{T-box Transcription Factor 6-associated Con- genital Scoliosis}

T-box transcription factor 6 (TBX6)-associated congenital scoliosis (TACS) is an example of genomic congenital scoliosis, since 
it has a significant impact on understanding the genetic basis of congenital scoliosis. TBX6 is located on chromosome 16p11.2, with 8 exons and 6,095 bp. This gene is a member of a phylogenetically conserved family of genes that share a common DNAbinding domain, the T-box. The T-box encodes transcription factors involved in the regulation of developmental processes. Knockout studies in mice indicate that this gene is important for the specification of paraxial mesoderm structures. TBX6 is also required for the segmentation of the paraxial mesoderm into somites and for the normal development of the dermomyotome in zebrafish. In the absence of TBX6, the central dermomyotome of zebrafish failed to develop.

TACS is a combination of a heterozygous 16p11.2 deletion/ TBX6 null variant and a trans hypomorph allele. In some Chinese cohorts, TACS accounts for approximately $10 \%$ of the congenital scoliosis. Chinese researchers reported that 31 out of 41 patients with TACS harbored a 16p11.2 deletion, while the other 10 carried TBX6 truncating alleles such as nonsense, frameshift, or canonical splicing site variants. In addition to the 16p11.2 deletion or TBX6 null variant, all 41 patients carried the T-(rs2289292), C-(rs3809624), A-(rs3809627) haplotype (frequency $=44.4 \%$, Chinese Han population) in trans with the $16 p 11.2$ deletion or null allele. From a clinical phenotypical perspective, all (41/41) of patients had hemivertebra or butterfly vertebra present at the lower half of the spine [15]. The 16p11.2 deletion affecting TBX6 or single-nucleotide variants, such as nonsense and frameshift mutations, is insufficient to cause congenital scoliosis. TACS shows the compound inheritance of a rare copy number variation and/or null mutation and a hypomorphic allele of TBX6, accounting for up to $11 \%$ of congenital scoliosis cases, in the Chinese cohort [21]. TBX6 missense variants expand the mutational spectrum in non-Mendelian inheritance disease. In this genetic model, the trait manifests as a result of the combined effect of a rare variant and a common pathogenic variant allele at a locus. Expanding the mutational spectrum of TBX6 pathogenic alleles enabled an increased molecular diagnostic detection rate, providing further evidence for the gene dosage-dependent genetic model underlying congenital scoliosis and refined clinical classification [22].

\section{EOS in Syndromes}

EOS can be found in several syndromes. When hypermobility is present (Beighton score $\geq 5 / 9$ points) in EOS, connective tissue disorders such as Marfan, Ehlers-Danlos, and Loeys-Dietz syndromes should be differentially diagnosed, and medical genomic evaluation is required. Therefore, three syndromes are reviewed in reference to the prevalence and pathogenesis of EOS.

\section{Neurofibromatosis type 1}

Neurofibromatosis type 1 (NF-1) is an autosomal dominant genetic disease that may involve multiple systems, including the skin, eyes, brain, and skeletal system [23,24]. NF-1 occurs in approximately one in 3,500 individuals and is classified as a RASopathy. Bone malformations include scoliosis, congenital arch and pseudo-joint formation, bone cyst, thinning of the cortical bone, and subperiosteal bone hyperplasia. Radiographs often show trabecula disruption, cortical distortion, sclerotic zone, bone hyperplasia, and soft tissue swelling [23]. Approximately $45 \%$ of the patients with NF-1 develop scoliosis or kyphoscoliosis. The deformity is rapidly progressive in the presence of dystrophic changes, that causes bone erosion. Vertebral subluxation or dislocation can occur in children with highly dystrophic kyphoscoliosis and carry an increased risk of paralysis. Patients with NF-1 can develop dystrophic kyphoscoliosis with segmental spinal subluxation/dislocation. The etiology of dystrophic scoliosis in NF-1 is as follows: mass effect from a neurofibroma, intrinsic bone dysplasia, osteopenia, dural ectasia leading to vertebral collapse, and neurofibroma tissue impacting the microenvironment of an intrinsically compromised osseous matrix, leading to scoliosis. Scoliosis is particularly problematic in NF-1 patients, with earlier onset, rapid progression, and morbidity [25].

\section{Noonan syndrome}

Noonan syndrome is a relatively common as it occurs in $1: 1,000$ to $1: 2,500$ live births. It is the second most frequent syndromic cause of congenital heart disease after Down syndrome. It is a genetically heterogeneous autosomal dominant malformation syndrome, and classified as a RASopathy. Proportionate short stature, dysmorphic face, and congenital heart defects constitute the characteristic features of this condition. Approximately $50 \%$ of the patients with Noonan syndrome have scoliosis, where scoliosis can be associated with osteopenia/osteoporosis frequently found in Noonan syndrome (about 14\%) and/or Chiari malformation type 1 [26-28].

\section{Prader-Willi syndrome}

PWS is a complex imprinting disorder caused by the lack of expression of paternally inherited genes on chromosome 15q11q13 with severe implications on endocrine, cognitive, and neurologic systems, metabolism, and behavior. The birth incidence of PWS ranges from 1/10,000 to 1/30,000. Orthopedic problems 


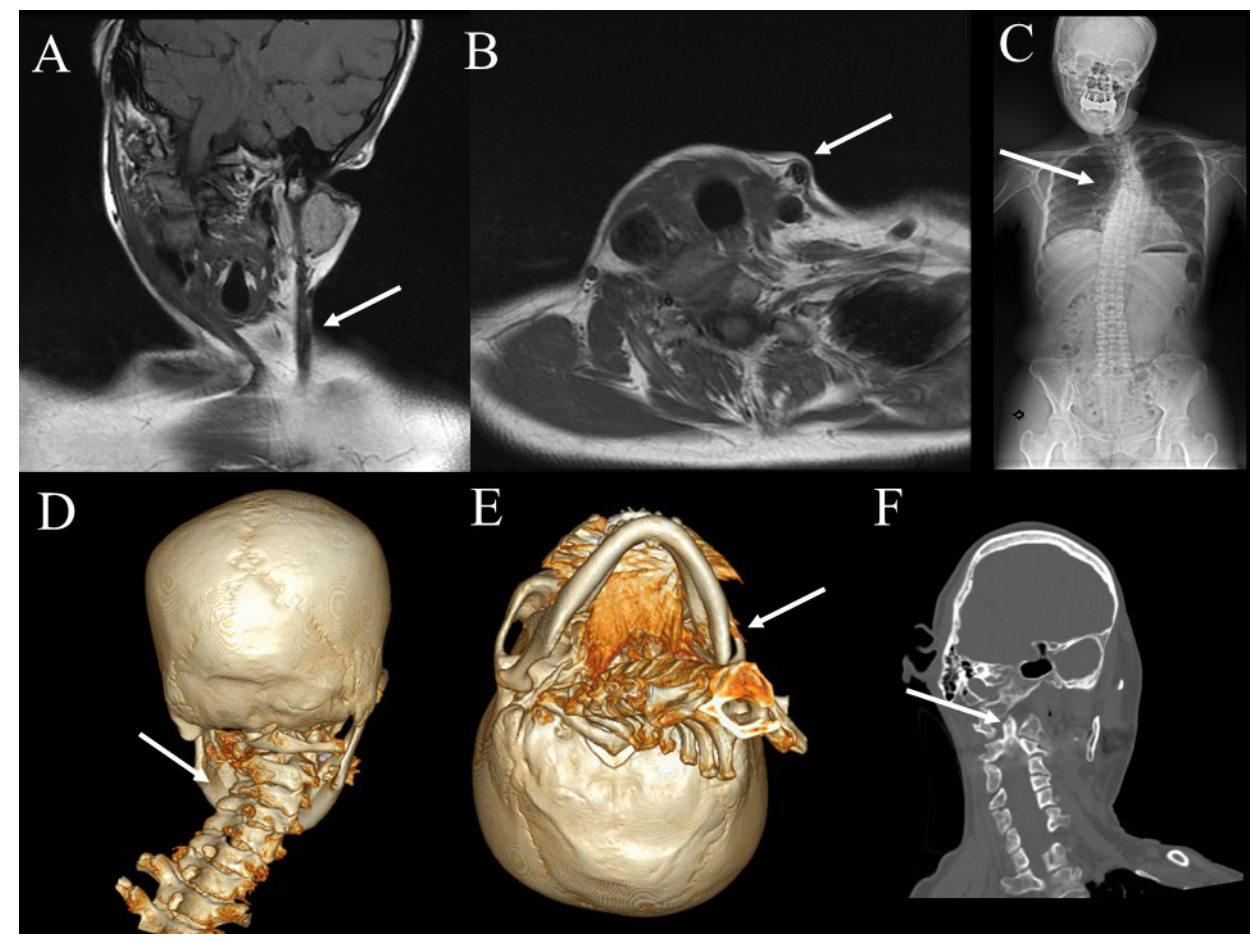

Fig. 3. A 52-year-old female with neglected left congenital muscular torticollis. Magnetic resonance imaging of the neck reveals short left sternocleidomastoid muscle (arrow, A) with low signal intensity in left sternocleidomastoid muscle (arrow, $B)$, suggesting left congenital muscular torticollis. The whole spine X-ray shows left thoracic scoliosis with $37.58^{\circ}$ of Cobb angle (arrow, C). Three-dimensional computed tomogram reveals left head tilting with $26.93^{\circ}$ of cervicomandibular angle (arrow, D), the facial asymmetry with flat left zygomatic arch (arrow, E), and craniovertebral junction abnormality with a shift of dense toward left (arrow, F). She underwent both unipolar resections of the left sternocleidomastoid muscle and post-operative physical therapy, showing improved symptoms. include scoliosis, which is bimodal with development within four years of age and later in childhood or early adolescence, with infantile spinal curvature. Approximately two-thirds of the patients with PWS are affected by scoliosis at the time of skeletal maturation. Most individuals with PWS have lumbar or thoracolumbar curves in contrast to thoracic curves in patients affected by idiopathic scoliosis without PWS [29]. While the underlying mechanisms of scoliosis in PWS are unknown, endocrine dysfunction such as hyperreninemia, growth hormone deficiency, and hypogonadism, could be related to the high prevalence of scoliosis in PWS [30,31].

\section{EOS in Neuromuscular Diseases}

While muscular dystrophy and cerebral palsy are the most common neuromuscular diseases causing EOS [32], scoliosis in muscular dystrophy and cerebral palsy is well studied. Congenital muscular torticollis is one of the most common neuromuscular EOS and Chiari malformation type I.

\section{Congenital muscular torticollis}

Congenital muscular torticollis is one of the most common skeletal problems in infants, found exclusively in neonates, and is characterized by a unilateral palpable neck mass of muscle consistency with significant limitation of passive rotation of the neck toward the shoulder of the shortened sternocleidomastoid muscle side. Neglected congenital muscular torticollis can cause EOS (Fig. 3). Therefore, congenital muscular torticollis should be included in the differential diagnosis of the underlying conditions of EOS.

\section{Chiari malformation type I}

Chiari malformation type I is one of the etiologies of EOS. Chiari malformation type I is a caudal displacement of the cerebellar tonsils through the foramen magnum, and descent of the cerebellar tonsil to at least $5 \mathrm{~mm}$ into the upper cervical canal [33]. The prevalence of Chiari malformation type I is $0.56 \%$ to $1 \%$ of the population [34]. The most common pathogenesis of Chiari malformation type I is a volumetric disproportion between the posterior fossa and its contents (reduced embryologic development of the skull base) with others such as hemodynamic/ cerebrospinal fluid dynamic alterations, resulting in increased intracranial pressure, mass effect within the posterior fossa (for example, tumors), and low intraspinal pressure due to craniospinal intrathecal pressure imbalance (for example, lumbo-peritoneal shunts). Clinical manifestations of Chiari malformation type I are related to arachnoid scarring and adhesions at the foramen magnum, possibly because of the rubbing of the cerebellar tonsils against the bone over many years. Arachnoid scarring and adhesions may also lead to the formation of syringomyelia [34]. Scoliosis in Chiari malformation type I is reported in up to $20 \%$ of the patients and in up to $60 \%$ of the patients with syringo- 
myelia [35]. Hypotheses on the occurrence of scoliosis in Chiari malformation type I are cerebellar tonsillar compression of the cervicomedullary junction and uneven expansion of a syrinx in the horizontal plane of the spinal cord [36,37]. Therefore, Chiari malformation type I should be included in the differential diagnosis of the underlying conditions of EOS.

\section{Conclusion}

I reviewed the etiology and pathogenesis of EOS in reference to medical genomics. The identification of the molecular etiology underlying patients with EOS has been increasing with advances in medical genomics. This provides valuable information for both medical management and screening.

\section{References}

1. Wise CA, Sepich D, Ushiki A, Khanshour AM, Kidane YH, Makki N, et al. The cartilage matrisome in adolescent idiopathic scoliosis. Bone Res 2020;8:13.

2. Jada A, Mackel CE, Hwang SW, Samdani AF, Stephen JH, Bennett JT, et al. Evaluation and management of adolescent idiopathic scoliosis: a review. Neurosurg Focus 2017;43:E2.

3. Cheng JC, Castelein RM, Chu WC, Danielsson AJ, Dobbs MB, Grivas TB, et al. Adolescent idiopathic scoliosis. Nat Rev Dis Primers 2015;1:15030.

4. Yang S, Andras LM, Redding GJ, Skaggs DL. Early-onset scoliosis: a review of history, current treatment, and future directions. Pediatrics 2016;137:e20150709.

5. Hasler CC. Early-onset scoliosis: contemporary decision-making and treatment options. J Pediatr Orthop 2018;38 Suppl 1:S13-20.

6. Thorsness RJ, Faust JR, Behrend CJ, Sanders JO. Nonsurgical management of early-onset Scoliosis. J Am Acad Orthop Surg 2015;23:51928.

7. Brockmeyer D. Congenital vertebral anomalies. [https://neupsykey. com/congenital-vertebral-anomalies/]

8. Riseborough EJ, Wynne-Davies R. A genetic survey of idiopathic scoliosis in Boston, Massachusetts. J Bone Joint Surg Am 1973;55:97482.

9. Pehrsson K, Larsson S, Oden A, Nachemson A. Long-term follow-up of patients with untreated scoliosis. A study of mortality, causes of death, and symptoms. Spine (Phila Pa 1976) 1992;17:1091-6.

10. Negrini S, Donzelli S, Aulisa AG, Czaprowski D, Schreiber S, de Mauroy JC, et al. 2016 SOSORT guidelines: orthopaedic and rehabilitation treatment of idiopathic scoliosis during growth. Scoliosis Spinal Disord 2018;13:3.
11. Weinstein $S L$, Dolan LA, Spratt KF, Peterson KK, Spoonamore MJ, Ponseti IV. Health and function of patients with untreated idiopathic scoliosis: a 50-year natural history study. JAMA 2003;289:559-67.

12. Burwell RG, Clark EM, Dangerfield PH, Moulton A. Adolescent idiopathic scoliosis (AIS): a multifactorial cascade concept for pathogenesis and embryonic origin. Scoliosis Spinal Disord 2016;11:8.

13. Williams BA, Matsumoto $H$, McCalla DJ, Akbarnia BA, Blakemore LC, Betz RR, et al. Development and initial validation of the Classification of Early-Onset Scoliosis (C-EOS). J Bone Joint Surg Am 2014;96:135967.

14. Williams BA, McClung A, Blakemore LC, Shah SA, Pawelek JB, Sponseller PD, et al.; Pediatric Spine Study Group. MRI utilization and rates of abnormal pretreatment MRI findings in early-onset scoliosis: review of a global cohort. Spine Deform 2020;8:1099-107.

15. Zhao $S$, Zhang $Y$, Chen W, Li W, Wang $S$, Wang $L$, et al.; Deciphering Disorders Involving Scoliosis and COmorbidities (DISCO) study. Diagnostic yield and clinical impact of exome sequencing in early-onset scoliosis (EOS). J Med Genet 2021;58:41-7.

16. Senkoylu A, Riise RB, Acaroglu E, Helenius I. Diverse approaches to scoliosis in young children. EFORT Open Rev 2020;5:753-62.

17. Mackel $C E$, Jada A, Samdani AF, Stephen JH, Bennett JT, Baaj AA, et al. A comprehensive review of the diagnosis and management of congenital scoliosis. Childs Nerv Syst 2018;34:2155-71.

18. Baker RE, Schnell S, Maini PK. A clock and wavefront mechanism for somite formation. Dev Biol 2006;293:116-26.

19. Musumeci G, Castrogiovanni $P$, Coleman $R$, Szychlinska MA, Salvatorelli $L$, Parenti $R$, et al. Somitogenesis: from somite to skeletal muscle. Acta Histochem 2015;117:313-28.

20. Chal J, Pourquié 0. Patterning and differentiation of the vertebrate spine. In: Pourquié O, ed. The skeletal system. Cold Spring Harbor (NY): Cold Spring Harbor Laboratory Press, 2009;41-115.

21. Wu N, Ming $X$, Xiao J, Wu Z, Chen $X$, Shinawi $M$, et al. TBX6 null variants and a common hypomorphic allele in congenital scoliosis. $\mathrm{N}$ Engl J Med 2015;372:341-50.

22. Chen W, Lin J, Wang L, Li X, Zhao S, Liu J, et al. TBX6 missense variants expand the mutational spectrum in a non-Mendelian inheritance disease. Hum Mutat 2020;41:182-95.

23. Tabata MM, Li S, Knight P, Bakker A, Sarin KY. Phenotypic heterogeneity of neurofibromatosis type 1 in a large international registry. $\mathrm{JCl}$ Insight 2020;5:e136262.

24. Mladenov KV, Spiro AS, Krajewski KL, Stücker R, Kunkel P. Management of spinal deformities and tibial pseudarthrosis in children with neurofibromatosis type 1 (NF-1). Childs Nerv Syst 2020;36:2409-25.

25. Tsirikos Al, Dhokia R, Wordie S. Rotatory dislocation of the spine in dystrophic kyphoscoliosis secondary to neurofibromatosis type 1. J Cent Nerv Syst Dis 2018;10:1179573518819484. 
26. Kyritsi EM, Kanaka-Gantenbein C. Autoimmune thyroid disease in specific genetic syndromes in childhood and adolescence. Front Endocrinol (Lausanne) 2020;11:543.

27. Smpokou P, Tworog-Dube E, Kucherlapati RS, Roberts AE. Medical complications, clinical findings, and educational outcomes in adults with Noonan syndrome. Am J Med Genet A 2012;158:3106-11.

28. Keh YS, Abernethy L, Pettorini B. Association between Noonan syndrome and Chiari I malformation: a case-based update. Childs Nerv Syst 2013;29:749-52.

29. Butler MG, Miller JL, Forster JL. Prader-Willi syndrome - clinical genetics, diagnosis and treatment approaches: an update. Curr Pediatr Rev 2019;15:207-44.

30. Tauber M, Coupaye M, Diene G, Molinas C, Valette M, Beauloye V. Prader-Willi syndrome: a model for understanding the ghrelin system. J Neuroendocrinol 2019;31:e12728.

31. van Bosse HJP, Butler MG. Clinical observations and treatment approaches for scoliosis in Prader-Willi syndrome. Genes (Basel)
2020;11:260.

32. Cheuk DK, Wong $V$, Wraige $E$, Baxter $P$, Cole $A$, N'Diaye $T$, et al. Surgery for scoliosis in Duchenne muscular dystrophy. Cochrane Database Syst Rev 2007;1:CD005375.

33. Wagner A, Grassner L, Kögl N, Hartmann S, Thomé C, Wostrack M, et al. Chiari malformation type I and basilar invagination originating from atlantoaxial instability: a literature review and critical analysis. Acta Neurochir (Wien) 2020;162:1553-63.

34. McVige JW, Leonardo J. Neuroimaging and the clinical manifestations of Chiari malformation type I (CMI). Curr Pain Headache Rep 2015;19:18.

35. Kelly MP, Guillaume TJ, Lenke LG. Spinal deformity associated with Chiari malformation. Neurosurg Clin N Am 2015;26:579-85.

36. Noureldine MHA, Shimony N, Jallo GI, Groves ML. Scoliosis in patients with Chiari malformation type I. Childs Nerv Syst 2019;35:1853-62.

37. Holly LT, Batzdorf U. Chiari malformation and syringomyelia. J Neurosurg Spine 2019;31:619-28. 